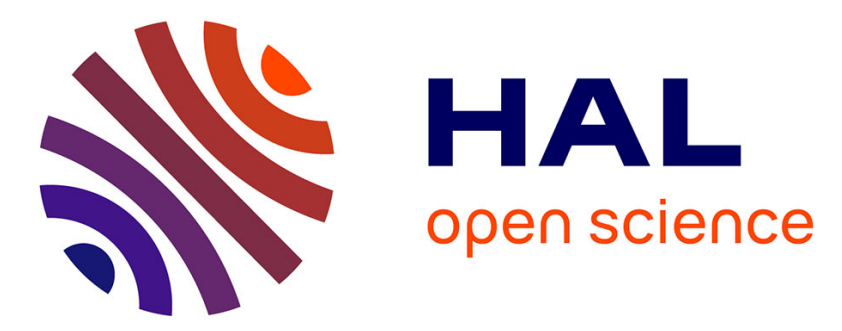

\title{
De multiples nodules abdominaux calcifiés chez une femme
}

Clémence Delteil, Kévin Caselles, Sophie Giusiano, Romain Appay, Aurélie Haffner, Nicolas Macagno

\section{To cite this version:}

Clémence Delteil, Kévin Caselles, Sophie Giusiano, Romain Appay, Aurélie Haffner, et al.. De multiples nodules abdominaux calcifiés chez une femme. Annales de Pathologie, 2020, 40 (1), pp.28-30. 10.1016/j.annpat.2019.12.003 . hal-03193894

\section{HAL Id: hal-03193894 \\ https://hal-amu.archives-ouvertes.fr/hal-03193894}

Submitted on 26 Apr 2021

HAL is a multi-disciplinary open access archive for the deposit and dissemination of scientific research documents, whether they are published or not. The documents may come from teaching and research institutions in France or abroad, or from public or private research centers.
L'archive ouverte pluridisciplinaire HAL, est destinée au dépôt et à la diffusion de documents scientifiques de niveau recherche, publiés ou non, émanant des établissements d'enseignement et de recherche français ou étrangers, des laboratoires publics ou privés.

\section{(ㅇ)(1) $\$$}

Distributed under a Creative Commons Attribution - NonCommercial - NoDerivatives| 4.0 


\title{
De multiples nodules abdominaux calcifiés chez une femme
}

\author{
Clémence Delteil ${ }^{\mathrm{a}, *, \mathrm{~b}}$, Kévin Caselles ${ }^{\mathrm{c}}$, \\ Sophie Giusiano ${ }^{c}$, Romain Appay ${ }^{b}$, Aurélie Haffner ${ }^{b}$, \\ Nicolas Macagno ${ }^{b}$
}

\author{
a Institut médico-légal de Marseille, hôpital de la Timone, Assistance publique-hôpitaux de \\ Marseille (AP-HM), 264, rue Saint-Pierre, 13005 Marseille cedex 5, France \\ b Inserm, anatomie pathologique et neuropathologie, MMG, université Aix Marseille, hôpital \\ de la Timone, AP-HM, 13005 Marseille, France \\ c Service d'anatomie et cytologie pathologiques, hôpital Nord, chemin des Bourrely, 13015 \\ Marseille, France
}

\section{Observation}

Une femme de 50 ans, sans antécédent notable, a bénéficié d'un scanner thoracique dans le cadre d'un bilan de dyspnée : le radiologue a alors découvert fortuitement de multiples formations centimétriques péritonéales confluentes, asymptomatiques, situées sous la coupole diaphragmatique gauche. La patiente ne présentait aucun signe d'appel clinique, ni d'altération de son état général. Une imagerie abdominale complémentaire par un scanner confirmait la présence de nombreux nodules de taille centimétrique, disposés dans la cavité abdominale, à l'étage sus-mésocolique. Un bilan plus poussé a été réalisé : la coloscopie et la fibroscopie œsogastroduodénale se révélaient sans particularité, l'examen gynécologique était normal, et le taux sanguin des marqueurs tumoraux ACE et CA 19/9 était normal. Suite à la négativité de ces explorations, et en raison de l'absence d'une piste diagnostique, l'indication d'une cœlioscopie exploratrice avec exérèse diagnostique a été posée. L'exploration peropératoire dénombrait une quarantaine de nodules, le plus volumineux mesurant $20 \mathrm{~mm}$ de grand axe. Un examen extemporané a été pratiqué. Son interprétation s'est révélée délicate : les coupes en congélation étaient techniquement très difficiles à réaliser, en raison de calcifications. L'examen microscopique des préparations d'extemporanée confirmait la présence de multiples psammomes, qui, en position abdominale, et dans les limites inhérentes à cet examen, ont été interprétées comme suspectes. 
L'examen histologique en coloration standard permettait une meilleure appréciation microsémiologique (Fig. 1) : il s'agissait de formations nodulaires, arrondies, bien limitées, non encapsulées, fibreuses, constituées d'une trame collagène hyaline, ponctuée de nodules lymphoïdes épars (Fig. 2A,B). De nombreuses calcifications arrondies, psam-

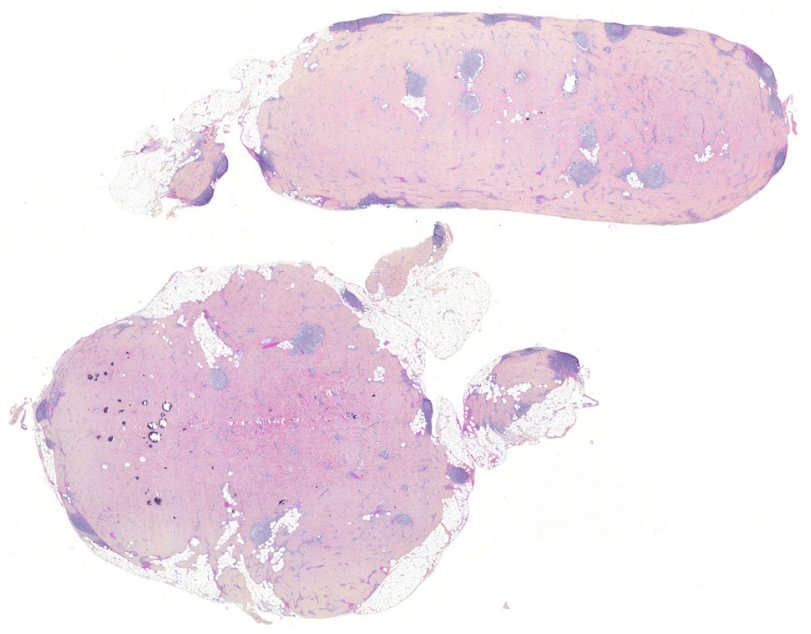

Figure 1. À ce grandissement $(25 \times)$, deux nodules fibreux sont observés, avec des calcifications, des foyers lymphoïdes et des amas d'adipocytes.

At this magnification (low-power view, $25 \times$ ), two fibrous nodules are observed, with calcifications, lymphoid aggregates and clusters of adipocytes.
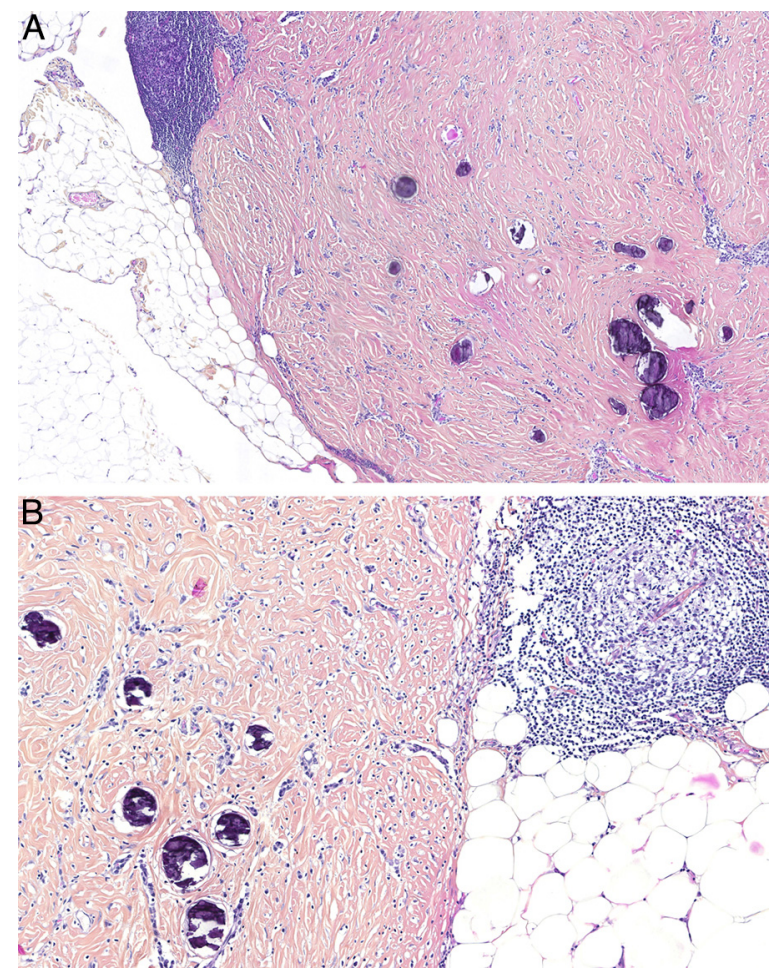

Figure 2. A, B. Les nodules sont hypocellulaires et sont constitués d'une abondante trame collagène d'aspect hyalin, de psammomes et de calcifications. Des amas lymphoïdes sont visualisés en périphéries, parfois centrés par un centre germinatif alors qu'un infiltrat interstitiel moins dense s'insinue entre les fibres de collagènes. The nodules are hypocellular and display an abundant hyaline collagenous core, psammomas and calcifications. Lymphoid aggregates are present at the periphery, sometimes with a germinal center. $A$ somewhat less cellular interstitial infiltrate is noted between the collagen bundles, thin capillaries are also visible. momateuses, étaient dispersées ainsi que quelques foyers lipogéniques matures. À plus fort grandissement, la trame conjonctive renfermait une population peu dense de cellules allongées, de cytologie fusiforme, fibroblastique, monotone et dépourvue d'atypie cyto-nucléaire ou d'activité mitotique, qui s'associait à un infiltrat plasmocytaire, de disposition interstitielle, réparti entres les nodules lymphoïdes. Cet infiltrat se présentait sous la forme de cellules isolées ou de petits groupements (Fig. 3). La vascularisation était peu marquée, constituée de petits capillaires à paroi fine. Aucun élément morphologique n'orientait vers une différenciation épithéliale, en particulier glandulaire. Le phénotype du contingent fusiforme était peu spécifique, avec expression du CD34 et du Facteur XIIla. Les immunomarquages dirigés contre la protéine $\mathrm{S} 100, \mathrm{AE} 1 / \mathrm{AE} 3$, calretinine, desmine, h-caldesmone, actinemusculaire-lisse (AML), ALK (5A4) et ROS étaient négatifs. Les hybridations in situ chromogéniques, complémentaires des transcrits des chaînes légères kappa et lambda, soulignaient le caractère polytypique de l'infiltrat plasmocytaire. Le ratio lgG4 sur CD138 était inférieur au seuil (40\%) évocateur d'une maladie associée aux IgG4.

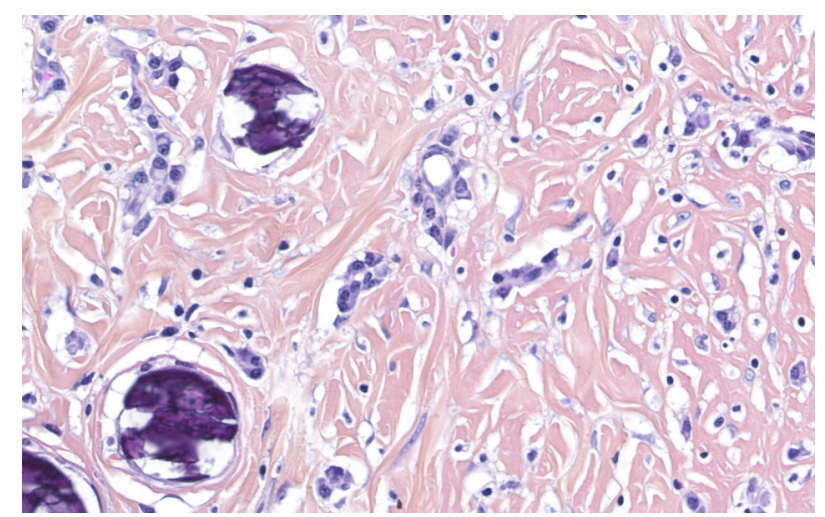

Figure 3. Fort grandissement, $400 \times$. L'infiltrat interstitiel est constitué de lymphocytes et de plasmocytes auquel s'associe à une prolifération de cellules fusiformes d'allure fibroblastique, qui est dépourvue de pléomorphisme, d'atypie ou d'activité mitotique. High-power view, $400 \times$. The interstitial infiltrate is composed of lymphocytes and plasma cells, admixed with fibroblastic spindle cells, without any pleomorphism, atypia or mitotic activity. 


\section{Diagnostic}

Tumeurs fibreuses calcifiantes multiples de l'abdomen.

\section{Discussion}

La tumeur fibreuse calcifiante a été décrite pour la première fois en 1988 par l'équipe de Rosenthal sous la dénomination de «tumeur fibreuse de l'enfance avec psamommes » [1]. Le terme actuellement retenu par la classification OMS des néoplasies des tissus mous est celui de tumeur fibreuse calcifiante [2]. Environ 150 cas ont été rapportés depuis.

La tumeur fibreuse calcifiante est une tumeur conjonctive rare et bénigne, qui est caractérisée par une silhouette nodulaire bien limitée, une population peu dense et non atypique de cellules de cytologie fusiforme, un stroma collagène hyalin, des foyers lympho-plasmocytaires et des calcifications, psammomateuses.

Tous les groupes d'âges peuvent être affectés, (de 5 mois à 84 ans, avec un âge médian de 43 ans pour les tumeurs viscérales [2] et de 38 ans pour les formes péritonéales [3]). Chez les enfants, les lésions apparaissent au niveau des tissus mous périphériques et profonds alors que chez l'adulte, elles surviennent préférentiellement au niveau des viscères. Les femmes sembleraient un peu plus affectées que les hommes, avec un sex-ratio de 1/1,27. Les localisations sont tout à fait ubiquitaires. Pour les localisations les plus fréquentes, et par ordre d'incidence, on peut citer l'estomac (18\%), l'intestin grêle $(8,7 \%)$, le péritoine $(6,8 \%)$ et le mésentère $(5 \%)$ [4]. Dans $95 \%$ des cas, ces lésions sont solitaires. Le caractère multifocal de ces lésions a été décrit, en particulier dans la région abdominale [4], à l'instar du cas que nous rapportons et qui peut poser un problème de diagnostic différentiel avec une carcinose péritonéale.

L'histoire clinique peut être longue et silencieuse : les tumeurs fibreuses calcifiantes sont souvent asymptomatiques (30\% des patients) ou pauci-symptomatiques [4] Le mode de révélation peut être fortuit, avec une découverte lors d'un bilan radiologique ou clinique d'une affection autre, comme dans le cas présenté [4]. La taille varie de $1 \mathrm{~mm}$ à $25 \mathrm{~cm}$, avec une médiane comprise entre 3,5 et $4,6 \mathrm{~cm}$, les lésions de plus grande taille surviennent préférentiellement au niveau du cou et de la glande surrénale $[4,5]$. Macroscopiquement, ces lésions sont nodulaires, bien limitées, parfois lobulées. À la coupe, elles présentent un aspect blanchâtre, homogène, avec un crissement caractéristique de la lame de dissection au contact des calcifications contenues dans ces lésions [4].

Le piège principal réside dans l'examen extemporané : la présence de psammomes en position abdominale chez une femme peut conduire le pathologiste à suggérer un diagnostic de carcinose péritonéale d'autant plus en cas de lésions multiples comme dans notre observation.

L'association d'une silhouette nodulaire, de collagène hyalin, de nodules lymphoïdes, d'un infiltrat plasmocytaire et de calcifications doit redresser le diagnostic qui peut être établi dès l'examen morphologique. Les diagnostics différentiels à discuter vont déprendre de la topographie des lésions et il faut, si la morphologie ou la présentation clinique est douteuse, éliminer les principales néoplasies (myo)fibroblastiques, inflammatoires ou présentant des calcifications : tumeur desmoïde, tumeur myofibroblastique inflammatoire, fasciite nodulaire, périneuriome, tumeur fibreuse solitaire, granulome calcifié, plaque pleurale calcifiée, lipome encapsulé, histiocytofibrome profond des tissus mous, mésothéliome desmoplastique, fibrome aponévrotique calcifiant et myosite ossifiante. S'il s'agit d'un nodule digestif viscéral, il faudra évoquer de principe une tumeur stromale gastro-intestinale (GIST). Selon la présentation clinique, une maladie associée aux IgG4, dans une forme pseudo-tumorale, doit aussi être discutée [6].

Une tendance à la diminution en taille de ces tumeurs, avec le temps, est décrite. Aucune donnée génomique ou cytogénétique n'est disponible sur cette entité : son histogenèse est incertaine. Une origine traumatique pour les cas adultes ou embryologique pour les cas pédiatriques, a été proposée [5].

Une récidive locale est décrite pour environ un dixième des patients, ce qui conforte l'idée d'un processus néoplasique plutôt qu'une pseudo-tumeur ou un processus fibreux séquellaire [4]. Ces tumeurs peuvent aussi être associées à des localisations (inclusions) ganglionnaires dans $7 \%$ des cas [5]. Le traitement est basé sur la chirurgie ou la surveillance. Le pronostic est excellent : à ce jour, aucun cas de métastase ou de décès en lien avec une tumeur fibreuse calcifiante n'a été rapporté, y compris dans les formes multiples.

\section{Déclaration de liens d'intérêts}

Les auteurs déclarent ne pas avoir de liens d'intérêts.

\section{Références}

[1] Rosenthal NS, Abdul-Karim FW. Childhood fibrous tumor with psammoma bodies. Clinicopathologic features in two cases. Arch Pathol Lab Med 1988;112:798-800.

[2] Nascimento AF. Calcifying fibrous tumour. In: Fletcher CDM, Bridge JA, Hogendoorn PCW, Mertens F, editors. WHO classification of tumours of soft tissue and bone. Fourth ed. Lyon: IARC Press; 2013. p. 321-4.

[3] Miettinen M, Fetsch JF, Antonescu CR, Folpe AL, Wakely Jr PE. AFIP atlas of tumour pathology. Tumors of the soft tissue. American Registry of pathology, Silver Spring; 2014.

[4] Chorti A, Papavramidis TS, Michalopoulos A. Calcifying fibrous tumor: review of 157 patients reported in international literature. Medicine (Baltimore) 2016;95:e3690.

[5] Kocova L, Michal M, Sulc M, Zamecnik M. Calcifying fibrous pseudotumour of visceral peritoneum. Histopathology 1997;31:182-4.

[6] Larson BK, Balzer B, Goldwasser J, Dhall D. Calcifying fibrous tumor: an unrecognized IgG4-related disease? APMIS 2015;123:72-6. 